\title{
Influencia de los juegos en la percepción del aprendizaje logrado y en la motivación a participar en clase: estudio de caso
}

José Antonio Garcia Arroyo

\begin{abstract}
Resumen
Introducción: El objetivo de esta investigación es estudiar de qué modo la aplicación de juegos en la materia de Planificación estratégica de recursos humanos de la Facultad de Ecología Humana, Educación y Desarrollo (de la Universidad Casa Grande/Ecuador) estimula y motiva a los alumnos a participar e interactuar en la clase y cómo influye en el aprendizaje de los mismos. Método: Durante los periodos lectivos de 2012 y 2013 los alumnos fueron expuestos a un juego que simula situaciones del ambiente real de trabajo mediante casos que ellos deberian resolver aplicando los conceptos relacionados con la materia. Se estimuló a los alumnos al trabajo en grupo y a participar y realizar actividades desafiantes en las clases. Se utilizó el cuestionario de evaluación que se usa de forma general en toda la universidad para medir la percepción sobre el aprendizaje logrado y la evaluación de la metodología empleada. Resultados: Los resultados muestran una relación positiva entre el uso de la metodología y el aprendizaje logrado tanto percibido por los alumnos como reflejados en sus calificaciones. Conclusiones: También se concluye que el uso de juegos estimula y motiva a los alumnos a trabajar en grupo, a participar más en la clase y a realizar actividades desafiantes, innovadoras e interesantes.
\end{abstract}

Palabras clave

Aprendizaje. Juegos educativos. Métodos de enseñanza..

\section{Introducción}

Este trabajo describe la aplicación de la metodología de aprendizaje basada en juegos y la metodología de casos en la materia de Planificación Estratégica de Recursos Humanos de la carrera de Gestión de Recursos Humanos de la Facultad de Ecología Humana, Educación y Desarrollo de la Universidad Casa Grande, mediante la implementación de un juego durante los años 2012 y 2013, y los resultados obtenidos a partir de la evaluación de los alumnos sobre dicha materia.

De acuerdo con Dempsey, Rasmussen y Lucassen (1996) los juegos estimulan el aprendizaje por que recrean un modelo de la realidad a través de una competencia y motivan a los participantes a jugar solo por el hecho de divertirse (Gómez Álvarez, 2010).

Los alumnos que toman la materia de Planificación estratégica de recursos humanos están en el cuarto año de su carrera y la mayoría de ellos trabaja durante el día. Esto hace que lleguen cansados a las clases lo que afecta a su rendimiento en el aula, les cuesta más concentrarse y tienden a participar menos, a permanecer pasivos y a no querer interactuar. Con esta base, se decidió incorporar una metodología dinámica e incluso lúdica, que favoreciera el aprendizaje jugando y que presentara actividades que estimularan y motivaran al alumno incluso a pesar del cansancio acumulado durante su jornada laboral. El principal objetivo de esta investigación fue estudiar de qué modo la aplicación de juegos en la materia de Planificación estratégica de recursos humanos estimula y motiva a los alumnos a participar e interactuar en la clase y cómo influye en el aprendizaje de los mismos. Para ello se plantearon las siguientes hipótesis:

Hipótesis 1: La aplicación de juegos en la materia de Planificación estratégica de recursos humanos estimula a los alumnos al trabajo en grupo y a 
participar y realizar actividades desafiantes en las clases.

Hipótesis 2: La aplicación de juegos en la materia de Planificación estratégica de recursos humanos aumenta el aprendizaje en los alumnos.

\section{Los juegos en educación}

Un juego se puede definir como una actividad interactiva que replica las condiciones esperadas en el mundo real, con el fin de estimular el aprendizaje en la toma de decisiones (Dempsey et al., 1996). Para lograr este fin, se plantea una competencia en la cual los participantes aceptan las reglas de conducta y toman decisiones que tienen implicaciones sobre sí mismos y sus contrincantes (Dempsey et al., 1996). Así mismo, un juego constituye un sistema formal cerrado que, subjetivamente, representa un subconjunto de la realidad (Crawford, 1984), lo que implica que cada vez que se ejecuta nuevamente se obtiene una nueva versión de la historia. Este resultado final depende de las condiciones globales del juego, así como de las características de sus participantes y su interacción (Kasvi, 2000).

Según Burgos, Tattersall y Koper (2006) para que un juego sea educativo debe tener las siguientes características: que parta de una premisa a resolver, que tenga por lo menos una solución cierta y que el jugador aprenda algo por medio de alguna técnica como la introducción de nuevos conocimientos; la fijación de conocimientos previamente adquiridos; el ejercicio de habilidades; el descubrimiento de conceptos; y el desarrollo de creaciones o la socialización de experiencia.

Además, los juegos educativos deben ser motivadores, es decir, generar entretenimiento en sus practicantes, quienes lo jugarán movidos por el deseo de divertirse (Jensen, 2006 citado por Gómez Álvarez, 2010). Deben ser capaces de simular una parte de la realidad, interactivos y dinámicos (Kasvi, 2000). En este caso en particular, los juegos basados en experiencias resultan bastante útiles pues, a diferencia de los juegos de computador, permiten una interacción directa del juga- dor con el entorno que se está simulando (Gee, 2003). Por último, los juegos deben ser seguros en el sentido de recrear una parte de la realidad pero sin ningún peligro para la salud o la integridad (Kasvi, 2000).

Utilizar juegos en los procesos de enseñanza-aprendizaje permite "aprender haciendo", desarrollar la comunicación, tener en cuenta el impacto de las emociones en el aprendizaje y estimular el aprendizaje por pares. También desarrollan el pensamiento crítico, la comunicación grupal, el debate y la toma de decisiones (Kober y Tarca, 2000; Zapata y Awad, 2007; Gómez Álvarez, 2010). Para Klassen y Willoughby (2003) los juegos incrementan la velocidad de aprendizaje y mejoran la retención y la memorización de conceptos. Además favorecen el aprendizaje experiencial porque proveen retroalimentación a las acciones que llevan a cabo los participantes, ya que les permiten ver las consecuencias de las decisiones tomadas (Fripp, 1997).

Entre los juegos más conocidos utilizados en procesos de enseñanza-aprendizaje están el juego de la cerveza (Senge, 1994), el juego de la confianza (Berg, Dickhaut y Mccabe, 1995), el juego para la enseñanza de la ética (Lloyd y Poel, 2008) y los juegos para decisiones tácticas (Crichton y Flin, 2001).

\section{Metodología}

La materia de Planificación estratégica de recursos humanos se dicta en el cuarto año de la carrera de Gestión de Recursos Humanos, en el que se supone que los alumnos ya conocen los diferentes subsistemas de gestión de recursos humanos como son el de incorporación de trabajadores a la empresa (selección, contratación e inducción), retención de los mismos (administración de retribuciones, formación y desarrollo) y desvinculación (evaluación, despido). Su objetivo es que los alumnos desarrollen habilidades de planificación estratégica aplicada a los diferentes subsistemas y teniendo en cuenta los objetivos estratégicos de la empresa, con el fin de tener la cantidad y calidad de trabajadores suficiente y necesaria en cada 
puesto de trabajo y en cada momento específico. Para ello, los alumnos deben aprender a analizar la cantidad de trabajadores que la empresa necesita en cada una de sus áreas y la preparación que éstos deben tener para que la empresa alcance sus objetivos estratégicos de la forma más eficiente posible. También deben saber qué herramientas usar y cómo usarlas para conseguir dichos objetivos. Cada periodo semanal de clase tiene una duración de tres horas y media, con un total de 48 horas en el semestre. La materia se imparte todos los años en el periodo de abril a julio.

\section{Descripción de la aplicación}

Se decidió implementar un juego llamado "El Director de Recursos Humanos" que combinara los elementos de estrategia y planificación aplicados a casos concretos de recursos humanos y en el que los alumnos tuvieran que trabajar en grupo. También se tuvo en cuenta el elemento lúdico y de azar propio de los juegos y de la vida misma.

El modelo está inspirado en el juego de Monopoli en el que hay un tablero (tabla de Excel) con diferentes casillas. Las casillas son de dos tipos: aquellas en las que el participante debe resolver un caso y aquellas en las que el participante debe desarrollar una habilidad.

Las casillas de casos consisten en que cuando un participante cae en dicha casilla se le plantea un caso sobre temas de planificación de recursos humanos. Se proponen simulaciones de situaciones del ambiente real de trabajo que el participante tiene que analizar, resolver y presentar una solución en la clase siguiente. Estas casillas de casos están ubicadas en el tablero en orden creciente de dificultad de manera que el participante, al ir avanzando en el juego tiene que enfrentarse con niveles de dificultad diferente.

Las casillas de habilidades consisten en actividades diseñadas para desarrollar las habilidades de análisis de información, planificación, evaluación y toma de decisiones, habilidades básicas que todo director de cualquier área de la empresa debe tener. Cuando un participante cae en estas casillas debe traer resuelta la actividad en la clase siguiente. Estas casillas están ubicadas en el tablero intercaladas entre las casillas de casos y aparecen con el nombre de "Sorpresa".

El tablero consta de 24 casillas de las cuales una es la salida, 19 son casillas de casos y 4 son casillas de habilidades. De los 19 casos, 13 son adaptaciones de los casos que presenta Puchol en su libro Nuevos Casos en dirección y Gestión de Recursos Humanos (Puchol, 2005). La adaptación se hizo teniendo en cuenta la realidad social y legal ecuatoriana. Los otros seis casos son creación propia. Tanto los casos como las actividades de habilidad son evaluados por el profesor sobre 10 puntos de manera que el alumno pueda tener una nota final que es el promedio de las notas obtenidas durante todo el juego.

Además del tablero, el juego de "el Director de Recursos Humanos" utiliza la aplicación llamada "Brain Trainer 2" (Mindscape, 2007) que es un video juego que desarrolla la habilidad verbal, numérica, espacial, lógica y de memoria con actividades en cinco diferentes niveles de dificultad para cada habilidad.

\section{Dinámica del juego}

La aplicación se implementó en los periodos de abril/julio de los años 2012 y 2013. En el periodo del 2012 hubo un total de quince alumnos y en el 2013 un total de nueve. El curso del año 2012 tenía una clase semanal (Martes) de tres horas y media con un descanso en la mitad de quince minutos, mientras que el del 2013 tenía dos periodos semanales (Martes y Jueves) de una hora y 45 minutos cada uno.

Al inicio del curso se explicó a los alumnos la metodología del juego y se agruparon a los alumnos en parejas. Para ello se selecciona al azar, lanzando un dado, la actividad de Brain Trainer 2 que los alumnos deben practicar. Con base en las puntuaciones obtenidas por los alumnos en esta actividad se seleccionan las parejas de tal modo que el alumno que obtuvo la mejor puntuación formará pareja con el de peor puntuación. El se- 
gundo de mejor puntuación con el segundo de peor puntuación y así sucesivamente. Este método intenta que las parejas estén equilibradas en cuanto a las habilidades de sus integrantes. Las parejas formadas serán las mismas para el resto del juego durante el curso. Después cada pareja elegirá una ficha de color que la identifique en el tablero.

Un miembro de cada pareja lanza un dado y mueve su ficha hasta la casilla correspondiente cuyo caso o habilidad deberá resolver. La clase siguiente comienza con la aplicación de Brain Trainer 2 en la cual participa un integrante de cada pareja, el que cada una decida. La actividad de Brain Trainer 2 a resolver se elige al azar, lanzando el dado. La pareja que obtiene la peor puntuación en esta actividad debe exponer ante sus compañeros la solución al caso que le había tocado en la clase anterior. Después el profesor aclara los fundamentos teóricos sobre la problemática que plantea el caso y da retroalimentación sobre la solución propuesta por los alumnos. El resto de parejas entrega al profesor la solución a sus casos de forma escrita, y éste a su vez les dará retroalimentación escrita.

Al final de la clase se vuelve a lanzar el dado para avanzar en el tablero y enfrentar un nuevo caso o habilidad. Durante el curso cada pareja debe tener al menos ocho notas entre resolución de casos y actividades de habilidad.

Al finalizar el curso los alumnos evaluaron la materia en cuanto al aprendizaje logrado y la metodología empleada. El instrumento para la recogida de los datos consistió en un cuestionario que es utilizado en la universidad para que los alumnos evalúen las materias y a los profesores al final de cada periodo académico con el objetivo de monitorear la calidad de la enseñanza, el cumplimiento de los programas académicos y el desempeño de los profesores. Por tanto no se construyó específicamente para medir los resultados de la aplicación de juegos en el proceso de aprendizaje, lo cual constituye una limitación de este estudio. Consta de tres partes: la primera (cinco ítems) evalúa la percepción del alumno sobre su aprendizaje con ítems como por ejemplo "He entendi- do, ampliado, mejorado o aclarado de qué se trata la materia"; en la segunda los alumnos evalúan la metodología empleada en las clases a través de ocho ítems como por ejemplo "Incluyó la realización de actividades de resolución de problemas profesionales o relativos a la vida en sociedad"; y en la tercera parte, que consta de ocho ítems, los alumnos evalúan al profesor. Para calificar se utiliza una Escala Likert con cinco niveles siendo 1 = "Casi nada" y 5 = "Muchísimo". La información de estas evaluaciones se solicitó a la Secretaría General de la Universidad. También se solicitaron las notas obtenidas por los alumnos en esta materia. Se pidió la información correspondiente a los años 2012 y 2013, años de implementación del juego y la del año 2011 para efectos de comparación.

\section{Resultados y discusión}

Se presentan en primer lugar los resultados de la evaluación de los alumnos sobre la percepción de su propio aprendizaje y las calificaciones (promedio de notas) que los alumnos obtuvieron (hipótesis 2). Después se presentan los resultados sobre la influencia de la metodología para estimular a los alumnos al trabajo en grupo y a participar y realizar actividades desafiantes en las clases (hipótesis 1).

Primero hay que señalar que no todos los alumnos que cursaron la materia en los años 2011 al 2013 la evaluaron (Tabla 1). En los años 2011 y 2013 el porcentaje de alumnos que evaluaron la materia es bajo. Esto puede ocurrir o bien porque al alumno se le olvidó evaluar o bien por que suspendió la materia y ya no quiere evaluarla.

Tabla 1 - Relación entre alumnos que cursan la materia y los que la evalúan

\begin{tabular}{lccc}
\hline & 2011 & 2012 & 2013 \\
\cline { 2 - 4 } Alumnos que cursaron la materia & 14 & 15 & 9 \\
Evaluaron la materia & 9 & 13 & 5 \\
& $64 \%$ & $87 \%$ & $56 \%$ \\
\hline
\end{tabular}

Fuente: Elaboración propia.

En cuanto a los resultados generales de las evaluaciones el gráfico 1 muestra que hay una correlación positiva alta $(r=.953)$ entre la percepción 
Gráfico 1 - Evaluación de la percepción de aprendizaje y la metodología aplicada

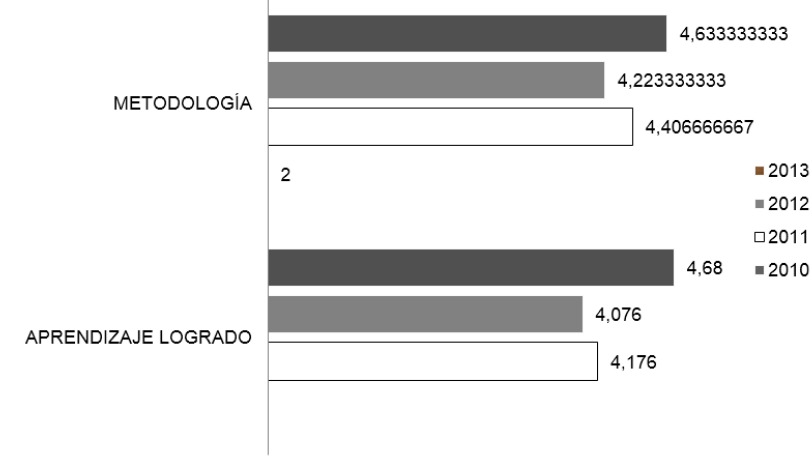

Fuente: Elaboración propia.

del aprendizaje y la evaluación de la metodología correspondiendo al año 2013 la puntuación más alta en ambas variables. También muestra que las puntuaciones más bajas corresponden al primer año de aplicación de la metodología basada en juegos, que fue el 2012.

Parecería que puede establecerse una relación entre ambas variables. Es decir, que una percepción positiva de la metodología empleada se relaciona con una percepción positiva sobre el aprendizaje logrado. Sin embargo, la influencia de variables como la cantidad y tipo de alumnos, diferentes todos los años, o los horarios de las clases, dos periodos semanales de una hora y 45 minutos en el 2011 y 2013 frente a un solo periodo de 3 horas y media en el 2012, pueden afectar estos resultados. Estas condiciones hacen que el resultado final sea variable y dependa de las condiciones globales del juego, así como de las características de los participantes y de su interacción como ya señaló Kasvi (2000). De hecho, el año 2012, primer año de la aplicación del juego en la materia se caracteriza por tener mayor cantidad de alumnos que los otros dos años, así como por su diferencia en cuanto a los periodos de clase y su duración. Tener dos periodos de clase de menor duración coincide con mejores calificaciones en las variables metodología y aprendizaje logrado.

En cuanto a la percepción sobre el aprendizaje logrado (Tabla 2), las puntuaciones de los alumnos en el primer año de aplicación de la metodología (2012) son inferiores en tres de los cinco ítems evaluados con respecto al año anterior. Sin embargo, en el segundo año de aplicación todas las puntuaciones son superiores. El promedio general de cada año indica que en el año 2012 la percepción sobre el aprendizaje logrado es menor que los otros dos años, pero en el año 2013 esta percepción es bastante superior. Esto podía indicar que la metodología de juegos hace que los alumnos aprendan más probando así la segunda hipótesis planteada en el estudio.

Tabla 2 - Percepción sobre el aprendizaje logrado

\begin{tabular}{|c|c|c|c|}
\hline Aprendizaje & 2011 & 2012 & 2013 \\
\hline $\begin{array}{l}\text { He entendido, ampliado, mejorado } \\
\text { o aclarado de qué se trata la } \\
\text { materia }\end{array}$ & 4,22 & 3,92 & 4,60 \\
\hline $\begin{array}{l}\text { Puedo realizar de mejor forma } \\
\text { trabajos y tareas que no podía } \\
\text { hacer al inicio del semestre, antes } \\
\text { de ver la materia }\end{array}$ & 4,33 & 3,92 & 4,80 \\
\hline $\begin{array}{l}\text { He aprendido a trabajar mejor, } \\
\text { reconocer aciertos y errores } \\
\text { de mis trabajos, buscar nuevas } \\
\text { soluciones, trabajar en equipo, ser } \\
\text { responsable con mis obligaciones }\end{array}$ & 4,33 & 4,08 & 4,60 \\
\hline $\begin{array}{l}\text { He mejorado o adquirido } \\
\text { habilidades para aprender por mi } \\
\text { cuenta }\end{array}$ & 4,00 & 4,08 & 4,60 \\
\hline \multirow[t]{2}{*}{$\begin{array}{l}\text { Tengo nuevas preguntas, } \\
\text { inquietudes o ganas de saber más } \\
\text { sobre algún tema que vimos en } \\
\text { el curso }\end{array}$} & 4,00 & 4,38 & 4,80 \\
\hline & 4,18 & 4,08 & 4,68 \\
\hline
\end{tabular}

Fuente: Elaboración propia.

Más específicamente parece que el uso de juegos desarrolla más las habilidades para el auto aprendizaje, estimula la capacidad para hacerse nuevas preguntas y las ganas de saber más.

Los resultados de las calificaciones obtenidas por los alumnos en esta materia en los años 2011 2013 (Tabla 3) muestran que los alumnos obtuvieron mejores resultados en sus notas en los dos años que se implementó la metodología de juegos. Esto también parece ser un argumento que apoye la segunda hipótesis planteada. Sin embargo, se observan diferencias entre la percepción de aprendizaje logrado y la nota obtenida en el año 2012. En este caso se constata una correla- 
ción negativa $(r=-0,465)$ entre las dos variables. Las diferencias entre los tres grupos de alumnos en cuanto a sus conocimientos previos, su capacidad para aprender y las relaciones entre los alumnos en cada grupo, podrían ser una explicación a estos resultados. Quizá la metodología de aplicación de juegos supone un desafío para los alumnos que les da una percepción de que aprenden más aunque a nivel de resultados, las notas sean más bajas. O quizá el aprendizaje logrado no logre representarse a través de una nota ya que la nota mide aprendizaje de tipo conceptual mientras que el aprendizaje logrado a través de juegos sea más de tipo procedimental y actitudinal (Kober y Tarca, 2000; Zapata y Awad, 2007). También debe tenerse en cuenta que los criterios de calificación de las actividades correspondientes a la metodología de juegos fueron distintos que los empleados en la metodología anterior, de manera que la comparación de las notas de estos años deba ser realizada con cierta precaución.

Tabla 3 - Calificaciones obtenidas en la materia

\begin{tabular}{lccc}
\hline & 2011 & 2012 & 2013 \\
\cline { 2 - 4 } Alumnos que cursaron la materia & 14 & 15 & 9 \\
Alumnos reprobados & 2 & 1 & 1 \\
Promedio de notas del curso & 8,59 & 8,98 & 8,67 \\
\hline
\end{tabular}

Fuente: Elaboración propia.

Por otra parte, el gráfico 2 muestra la evaluación de los años 2011 a 2013 que los alumnos hicieron sobre la metodología empleada. En general, la evaluación de la metodología en el 2013 tiene puntuaciones más elevadas que en los otros dos años. A su vez, la evaluación del año 2012 arroja puntuaciones inferiores respecto de las del 2011 en algunos ítems excepto en aquellos que hacen referencia a la aplicación de actividades interesantes, innovadoras y desafiantes, características que deben tener los juegos educativos de acuerdo con Gómez Álvarez (2010), y a la participación en actividades grupales. Así mismo, los ítems que obtuvieron puntuaciones más altas en la evaluación del 2013 reflejan las características de los juegos educativos como generar frecuentes oportunidades para compartir las ideas, conocimientos y opiniones de otros compañeros de curso, ya sea en trabajos en grupos, presentaciones o talleres en clases (puntuación 4.8) (Burgos, Tattersall y Koper, 2006; Gómez Álvarez, 2010); exigir a los alumnos hacer trabajos individualmente, que les ayudaron a entender mejor la materia por si mismos (puntuación 4.8) (Dempsey et al., 1996); hacerles pensar sobre cómo y qué han aprendido a través del curso (puntuación 4.8) (Fripp, 1997). Estos resultados dan razón de la primera hipótesis de estudio planteada según la cual la aplicación de juegos en la materia de Planificación estratégica de recursos humanos estimula a los alumnos al trabajo en grupo y a participar y realizar actividades desafiantes en las clases.

Gráfico 2 - Evaluación de la metodología

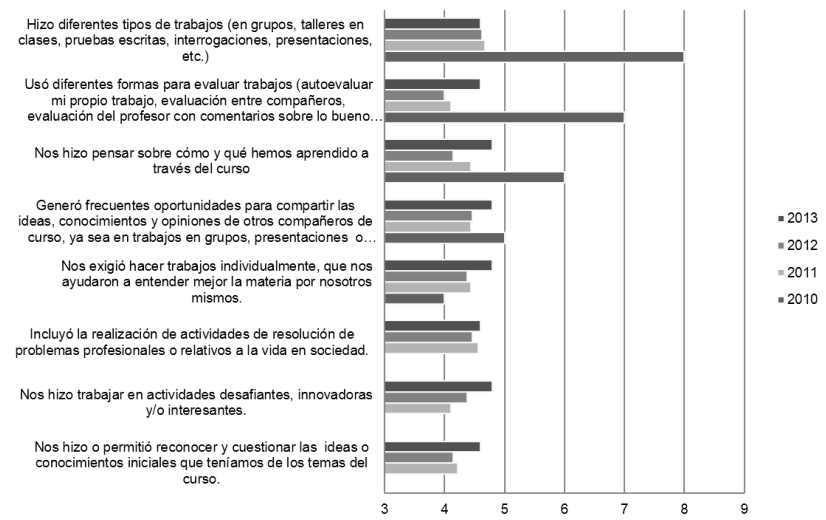

Fuente: Elaboración propia.

\section{Conclusiones}

La aplicación de la metodología del uso de juegos en el proceso de enseñanza- aprendizaje produjo los resultados esperados: a través de los juegos los estudiantes están más motivados a participar en las cases y a trabajar en actividades desafiantes, innovadoras e interesantes y tienen la percepción de haber aprendido más. Esto concuerda con lo ya señalado por algunos autores (Dempsey et al., 1996; Gómez Álvarez, 2010) sobre los beneficios de emplear juegos en educación.

También parece que hay una correlación positiva entre el uso de la metodología de juegos y el incremento del aprendizaje logrado, lo que sugiere que la aplicación de juegos contribuye a un mayor aprendizaje no solo a nivel de percepción del alumno sino también a nivel de resultados en sus notas. Sin embargo esta conclusión debe ser tomada con precaución ya que la influencia de va- 
riables como tamaño del grupo, tipo de alumnos y conocimientos previos de los mismos, duración de los períodos de clase, entre otras también son importantes. Por ejemplo, a la vista de los resultados, podría decirse que en cuanto a la duración de los periodos semanales de clase es más aconsejable que sean dos más cortos en vez de uno solo más largo. En este estudio no se ha considerado la influencia de estas variables ya que la experiencia se realizó en años diferentes y con grupos de alumnos diferentes. Sería interesante poder realizar estudios de aplicación de juegos que controlen estas condiciones para tener una idea más aproximada de la influencia de la aplicación de juegos sobre el aprendizaje.

El hecho de que algunas calificaciones, como la del 2013, no reflejen la percepción de los estudiantes de que aprendieron más puede estar relacionada con el tipo de aprendizaje que facilita el uso de juegos. Aunque según Klassen y Willoughby (2003) los juegos mejoran la retención y la memorización de conceptos, también es cierto que desarrollan el pensamiento crítico, la comunicación grupal, el debate y la toma de decisiones (Gómez Âlvarez, 2010), competencias que no son medidas a través de una nota. A su vez, el aspecto motivador y lúdico del juego pude crear en el participante la sensación de haber aprendido más. Como señala D. Kirkpatrick y J. Kirkpatrick (2007) "una reacción positiva puede no asegurar el aprendizaje, pero una reacción negativa, casi con toda certeza reduce la posibilidad de que ocurra” (p. 44).

Una limitación del estudio está en haber utilizado la evaluación genérica diseñada para todas las materias de la universidad independientemente de la metodología que usen y no haber diseñado un instrumento de medición específico para la aplicación de juegos educativos. Aunque también hay que señalar que el instrumento utilizado se creó basado en la metodología de aprender haciendo, propia de la Universidad Casa Grande, y que los juegos educativos estimulan este tipo de aprendizaje como señalan Kober y Tarca (2000). Por último, este estudio puede abrir el campo para futuras investigaciones sobre el aprendizaje basado en juegos en la misma o en otras materias de la universidad. Por ejemplo, se puede ver el efecto de los juegos sobre el aprendizaje incluyendo un grupo de control estableciendo condiciones similares en ambos grupos. O se puede ver el efecto de los juegos sobre el desarrollo de competencias específicas. La metodología de enseñanza de la Universidad Casa Grande se fundamenta en el "aprender haciendo" y esto, como señala Gómez Álvarez (2010) es uno de los elementos centrales del uso de juegos en educación.

\section{Referências}

Berg, J., Dickhaut, J.. \& Mccabe, K. (1995). Trust, reciprocity, and social history. Games and Economic Behavior, 10(1), 122-142.

Burgos, D., Tattersall, C., \& Koper, R. (2006). Can IMS Learning Design be used to model computerbased educational games? BINARIA Magazine, (5). Recuperado en 9 de agosto de 2013, de http://dspace. ou.nl/bitstream/1820/673/1/BINARIA_Issue5_burgos_ et_al.pdf.

Crawford, C. (1984). Art of Computer Game Design. Estados Unidos: McGraw Hill.

Crichton, M., \& Flin, R. (2001). Training for emergency management: tactical decision games. Journal of Hazardous Materials, 88(2-3), 255-266. Recuperado en 2 de noviembre de 2013, de doi:10.1016/S03043894(01)00270-9.

Dempsey, J., Rasmussen, K., \& Lucassen, B. (1996). The instructional gaming literature: implications and 99 Sources. College of Education: University of South Alabama. (COE Technical Report No. 96-1). Recuperado en 2 de noviembre de 2013, de http://citeseerx.ist.psu.edu/viewdoc/ download?doi=10.1.1.202.2287\&rep=rep1\&type=pdf.

Gee, J. (2003). What Video Games Have to Teach Us About Learning and Literacy. New York: Palgrave Macmillan.

Gómez Álvarez, M. (2010). Definición de un método para el diseño de juegos orientados al desarrollo de habilidades gerenciales como estrategia de entrenamiento empresarial. Universidad Nacional de Colombia. Recuperado 3 agosto de 2013, de http:// www.bdigital.unal.edu.co/1968/.

Fripp, J. (1997). A future for business simulations? Journal of European Industrial Training, 21(4), 138-142. Recuperado 2 de noviembre de 2013, de doi:10.1108/03090599710171387. 
Kasvi, J. (2000). Not just fun and games: internet games as training medium. In P. Kymäläinen \& L. Seppanen (Eds.), Cosiga - Learning With Computing Simulation, (pp.22-33). HUT: Espoo.

Kirkpatrick, D., \& Kirkpatrick, J. (2007). Evaluación de acciones formativas los cuatro niveles. Barcelona: Ediciones Gestión 2000.

Klassen, K., \& Willoughby, K. (2003). In-class simulation games: assessing student learning. Journal of Information Technology Education, (2), 1-13.

Kober, R., \& Tarca, A. (2000). For fun or profit? an evaluation of a business simulation game. Accounting Research Journal, 15(1), 98-110. Recuperado en 2 de noviembre de 2013, de http://www.scholr.ly/ paper/1962339/for-fun-or-profit-an-evaluation-of-anaccounting-simulation-game.

Lloyd, P., \& Poel, I. (2008). Designing games to teach ethics. Sci Eng Ethics, 14(3), 433 - 447. Recuperado en 2 de noviembre de 2013, de doi: 10.1007/s11948008-9077-2.

Mindscape. (2007). Brain Trainer (Versión 2) [Computer Software]. Editorial Planeta DeAgostini Interactive.

Puchol, L. (2005). Nuevos Casos en dirección y Gestión de Recursos Humanos. Madrid: Editorial Diaz de Santos.

Senge, P. (2004). The fifth discipline: the art and practice of the learning organization. New York: Currency Doubleday.

Zapata, C., \& Awad, G. (2007). Requirements game: teaching software projects management. CLEI Electronic Journal, 10(1). Recuperado en 2 de noviembre de http://www.clei.org/cleiej/papers/ v10i1p3.pdf.
Influence of games in the perception of learning achievement and the motivation to participate in class: a case study

\begin{abstract}
Introduction: The objective of this research is to study how the gaming application on the subject of human resource strategic planning taught by the Faculty of Human Ecology, Education and Development (at the University Casa Grande/ Ecuador) encourages and motivates students to participate in the class activities and how games affect their learning. Method: During the class periods of 2012 and 2013 students were exposed to a game that simulates real environmental work situations they had to resolve by applying the concepts related to the subject. Students were encouraged to work in groups and participate in challenging activities in classes. The evaluation questionnaire generally used throughout the university to measure the perceptions of the learning achieved and evaluation methodology was applied. Results: The results show a positive relationship between the use of the methodology and learning achieved, not only perceived by the students but also reflected in their grades. Conclusions: It is also concluded that the use of games encourages and motivates students to work in groups, to participate actively in class and to perform challenging, innovative and interesting activities.
\end{abstract}

\section{Keywords}

Learning. Educational games. Teaching methods.

Recibido: Septiembre 25, 2013

Aceptado: Noviembre 1, 2013

\section{Acerca de los autores:}

\section{José Antonio Garcia Arroyo}

Licenciatura en Filosofia - Universidad de Murcia/Espanha, Máster em Dirección de Recursos Humanos - UNED/Espanha, Doctorando en Psicología Social y de las Organizaciones - Facultad de Psicología, UNED/Espanha. Professor investigador - Facultad de Ecología Humana, Educación y Desarrollo/UCG.

jgarcia@casagrande.edu.ec

Para citar el artículo:

GARCIA ARROYO. J. A. Influencia de los juegos en la percepción del aprendizaje logrado y en la motivación a participar en clase: estudio de caso. AtoZ: novas práticas em informação e conhecimento, Curitiba, v. 2, n. 2, p. 8693, jul./dez. 2013. Disponivel em: <http://www.atoz.ufpr. br>. Acesso em: 